\title{
Energetics of Propellant Options for High-Power Hall Thrusters
}

\author{
Alex Kieckhafer* and Lyon B. King $\ddagger$ \\ Michigan Technological University, Houghton, Michigan 49931
}

DOI: $10.2514 / 1.16376$

\begin{abstract}
Krypton, cadmium, iodine, cesium, mercury, and bismuth are compared with xenon in several areas of performance, including thrust, specific impulse, probability of ionization, maximum theoretical efficiency, and sputter yield. The lighter propellants such as krypton and cadmium are favorable for high-I $I_{\text {sp }}$, low-thrust applications, whereas heavier propellants such as mercury and bismuth are preferable for low- $I_{\text {sp }}$, high-thrust missions. Calculations of the ionizing collision rate show that cesium had the highest ionization probability (and lowest ionization energy), krypton had the lowest probability (and highest ionization energy), with the other propellant falling between cesium and krypton. Sputter erosion calculations show that for a carbon surface, heavier atoms will sputter less at low ion energies (less than $2000 \mathrm{eV}$ ) than light atoms, and will sputter much less on a kilograms-per-kilogram basis.
\end{abstract}

\section{Nomenclature}

$E_{i} \quad=$ Ion energy, $\mathrm{eV}$

$E_{\text {ion }} \quad=$ ionization energy, $\mathrm{J} / \mathrm{kg}$

$E_{\mathrm{th}} \quad=$ sputtering threshold energy, $\mathrm{eV}$

$F \quad=$ thrust, $\mathrm{N}$

$g \quad=$ gravitational acceleration, $9.8 \mathrm{~m} / \mathrm{s}^{2}$

$I_{\mathrm{sp}} \quad=$ specific impulse, $\mathrm{s}$

$k=$ Boltzmann constant, $1.38 \times 10^{-23} \mathrm{~m}^{2} \cdot \mathrm{kg} \cdot \mathrm{s}^{-2} \cdot \mathrm{K}^{-1}$

$L=$ discharge chamber length, $\mathrm{m}$

$M_{i} \quad=$ ion mass, $\mathrm{kg}$

$M_{n} \quad=$ mass of species $n, \mathrm{~kg}$

$M_{s} \quad=$ atomic mass of surface material, $\mathrm{kg}$

$M_{\mathrm{Xe}} \quad=$ mass of a xenon atom, $\mathrm{kg}$

$\dot{m} \quad=$ mass flow rate, $\mathrm{kg} / \mathrm{s}$

$n_{e} \quad=$ electron density, $\mathrm{m}^{-3}$

$P_{\text {ion }} \quad=$ power required for propellant ionization, $\mathrm{W}$

$P_{\text {kin }}=$ exhaust kinetic power, $\mathrm{W}$

$Q_{s} \quad=$ surface sputtering parameter

$q_{i} \quad=$ ion charge, $\mathrm{C}$

$S_{e}(\epsilon)=$ reduced Lindhard electronic stopping cross section, $\mathrm{m}^{2}$

$S_{n}(\epsilon)=$ nuclear stopping cross section, $\mathrm{m}^{2}$

$T=$ temperature, $\mathrm{K}$

$t_{r} \quad=$ residence time, $\mathrm{s}$

$U_{0} \quad=$ surface binding energy, $\mathrm{eV}$

$u_{e} \quad=$ exhaust velocity, $\mathrm{m} / \mathrm{s}$

$\left(u_{e}\right)_{\mathrm{Xe}}=$ velocity of xenon, $\mathrm{m} / \mathrm{s}$

$V_{a}=$ effective acceleration voltage, $\mathrm{V}$

$\bar{v}_{e} \quad=$ average electron velocity, $\mathrm{m} / \mathrm{s}$

$\bar{v}_{n} \quad=$ average neutral atom velocity, $\mathrm{m} / \mathrm{s}$

$Y_{E} \quad=$ sputter yield, atoms/ion

$\alpha_{s} \quad=$ surface sputtering parameter

$\gamma \quad=$ elastic energy transfer factor

$\epsilon=$ reduced energy parameter, $\mathrm{eV}$

$v \quad=$ ionizing collision frequency, $\mathrm{Hz}$

$\bar{\sigma}=$ average electron-impact ionization cross section, $\mathrm{m}^{2}$

Received 2 March 2005; revision received 20 January 2006; accepted for publication 3 March 2006. Copyright (C) 2006 by Alexander W. Kieckhafer. Published by the American Institute of Aeronautics and Astronautics, Inc., with permission. Copies of this paper may be made for personal or internal use, on condition that the copier pay the $\$ 10.00$ per-copy fee to the Copyright Clearance Center, Inc., 222 Rosewood Drive, Danvers, MA 01923; include the code $\$ 10.00$ in correspondence with the CCC.

${ }^{*}$ Graduate Research Assistant, 1400 Townsend Drive. Student Member AIAA

${ }^{\dagger}$ Assistant Professor, 1400 Townsend Drive. Member AIAA.

\section{Introduction}

A $\mathrm{N}$ emerging hurdle in high-power Hall thruster development has been the use of xenon as the propellant of choice. Whereas xenon has several advantages as a propellant, namely, low ionization energy, high atomic mass and easy storage and flow metering, several disadvantages preclude the use of xenon in very high-power thrusters. The first disadvantage to xenon as an electric propulsion (EP) propellant is its high cost. Currently xenon can be purchased for approximately $\$ 6.65$ per standard liter $(\$ 1140 / \mathrm{kg})$ in small quantities.. Using current commercial prices, a $500-\mathrm{kW}$ Hall thruster operating at $60 \%$ anode efficiency and $2000 \mathrm{~s}$ specific impulse will consume $\$ 6400$ of xenon per hour of operation. These costs can be extrapolated to $\$ 153,600$ per test day, and $\$ 64$ million for a 10,000hour mission. Longer-duration missions using larger thrusters or many smaller thrusters can quickly become relatively expensive to supply with propellant. To reduce this cost, more economical propellants need to be used. Studies have focused on krypton as a more economical alternative to xenon, while maintaining the general design of a gas-propelled thruster [1].

The second major disadvantage to xenon is in ground testing. Thruster exhaust must be evacuated from a test facility to maintain a spacelike vacuum. Typically, this is accomplished with cryogenic vacuum pumps. High facility pressures will affect the thruster performance and beam characteristics through an increase in chargeexchange ions created by collisions between exhaust ions and neutral atoms that remain the vacuum chamber. For a $500-\mathrm{kW}$ thruster operating at $2000 \mathrm{~s} I_{\mathrm{sp}}$ and $60 \%$ efficiency, $1.6 \mathrm{~g} / \mathrm{s}$ of xenon will enter the chamber. To maintain a pressure of $1 \times 10^{-5}$ Torr $\left(6.7 \times 10^{-4} \mathrm{~Pa}\right)$ of xenon, the facility vacuum pumps must be capable of nearly $20 \times 10^{6}$ liters per second of pumping throughput. The pressure chosen was previously determined by Randolph to be sufficient to nearly eliminate facility backpressure effects. More recent observations [2] have shown this not to be entirely accurate; however, for lack of another estimate of facility pressure, the estimate of Randolph will be used. At a cost of roughly $\$ 1$ per liter/s, $\mathrm{s}$ this translates to roughly $\$ 20$ million in pumping equipment. The pumping requirements scale linearly with thruster power, so a 1 MW thruster will require nearly $\$ 40$ million in pumping equipment. Additional costs include the large vacuum chamber, support infrastructure and recurring costs such as liquid nitrogen. Unlike propellant costs, facility costs cannot be reduced unless gaseous propellant usage is eliminated, as any gaseous propellant will require evacuation from the facility.

Fraxair, Inc., verbal quotation, http://www.praxair.com [cited June 2005].

§PHPK, Inc., verbal quotation for TM-1200 model cryopump, http:// www.phpk.com [cited June 2005]. 
Table 1 Physical properties of candidate propellants

\begin{tabular}{|c|c|c|c|c|c|}
\hline Propellant & Melting point, ${ }^{\circ} \mathrm{C}$ & Boiling point, ${ }^{\circ} \mathrm{C}$ & First ionization energy, eV & Atomic mass, amu & Cost per kg \\
\hline Bismuth (Bi) & 271 & 1559 & 7.3 & 209.0 & $\$ 6 \mathrm{a}$ \\
\hline Cadmium (Cd) & 321 & 765 & 9.0 & 112.4 & $\$ 25 \underline{b}$ \\
\hline Cesium (Cs) & 29 & 685 & 3.9 & 132.9 & $\$ 40,000 \underline{\mathrm{c}}$ \\
\hline Krypton (Kr) & -157 & -153 & 14.0 & 83.8 & $\$ 295$ e \\
\hline Mercury (Hg) & -39 & 357 & 10.4 & 200.6 & $\$ 4 f^{f}$ \\
\hline Xenon $(\mathrm{Xe})$ & -112 & -108 & 12.1 & 131.3 & $\$ 1138$ e \\
\hline
\end{tabular}

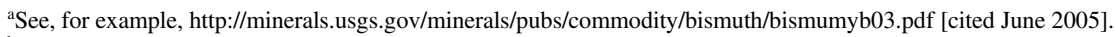

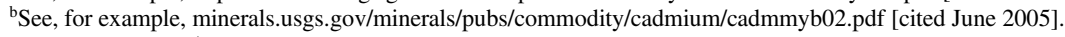

${ }^{c}$ Catalog price 198/5 g 99.999\% purity, from Sigma-Aldrich, Inc., www.sigmaaldrich.com [cited June 2005].

${ }^{\mathrm{d} C}$ Catalog price 242/500 g USP, from Sigma-Aldrich, Inc., www.sigmaaldrich.com [cited June 2005].

ePraxair, Inc., verbal quotation, www.praxair.com [cited June 2005].

${ }^{\mathrm{f}} \mathrm{See}$, for example, http://minerals.usgs.gov/minerals/pubs/commodity/mercury/430798.pdf [cited June 2005].

Condensible propellants, defined as those species existing in either solid or liquid state at standard temperature and pressure $\left(\mathrm{STP}, 0^{\circ} \mathrm{C}\right.$ and 1 atm pressure) offer significant advantages for facility cost. Whereas xenon, krypton, and other gaseous propellants must be actively evacuated from the test chamber, condensible propellants will naturally condense on the chamber walls, requiring no pumping. Thus, condensible propellants are "self-pumping," requiring only enough vacuum pump capacity to reach and maintain high vacuum with little to no gas load. Condensible propellants may also cost less than xenon or krypton, reducing testing costs further. Vacuum facility contamination may be an issue with condensable propellants, however. The coating of interior surfaces with propellant may require special handling procedures, especially for toxic and reactive propellants.

\section{Analysis of Alternative Propellants}

Many factors contribute to the suitability of a Hall thruster propellant. Factors that affect performance include ionization energy, atomic mass, and ease of mass flow system construction. Condensible propellants offer a challenge in mass flow systems, as traditional gas-fed systems used on xenon and krypton thrusters cannot be used. Generally, the higher the melting and boiling points of a propellant, the harder it will be to design and fabricate a mass flow system. Propellant ionization is another source of inefficiency. As energy spent on ionization is not available for acceleration, reduction of ionization energy will directly increase the efficiency of a thruster. Several practical issues also contribute to the suitability of a candidate propellant. These include cost, toxicity/reactivity, and potential for spacecraft contamination. Reducing propellant cost is of obvious benefit, as less-expensive propellants allow for reduced testing and mission costs. Several alternative propellant options have been considered [3-8]. Some physical properties of these propellants, including xenon as a point of comparison, are in Table 1. This section will discuss the relative merits and difficulties with the many propellant alternatives.

Several of the Hall thruster propellants considered are toxic and/or reactive. These include cadmium, $\mathbb{\|}$ cesium, $\mathbb{I}$ iodine,,$*$ and mercury.. \pm Whereas each of these propellants offer lower propellant costs and ionization energy than xenon, they all present problems in a testing environment. Of these, iodine and cesium present significant reactivity hazards, whereas each presents a significant chronic and/or acute toxicity hazard. Currently there are no active development efforts on these propellants.

Three of the propellants considered are relatively nontoxic and nonreactive. Xenon and krypton present essentially no toxicity threat

\footnotetext{
"Data available on-line at http://www.osha.gov/SLTC/cadmium [cited June 2005].

[Data available on-line at http://www.espi-metals.com/msds's/cesium.htm [cited June 2005].

**Data available on-line at http://www.jtbaker.com/msds/englishhtml/ i2680.htm [cited June 2005].

thata available on-line at http://www.jtbaker.com/msds/englishhtml/ M1599.htm [cited June 2005].
}

except through displacement of oxygen. $\$$ Reactivity is also of little concern, as krypton will not spontaneously react with any substance, and xenon will react only with fluorine. Bismuth presents little hazard. It is considered mildly toxic and safety precautions involve exposure limitation only. $\underline{\underline{\&}}$ Whereas conversion of a Hall thruster from xenon to krypton requires trivial redesign of the mass flow system, bismuth presents significant development issues due to its condensable nature. Bismuth thruster development is under consideration at several research laboratories $[\underline{9,10]}$.

\section{Energetics of Propellant Alternatives}

\section{A. Acceleration Kinetics}

The most obvious disparity between propellant performance characteristics is a change in thrust under identical discharge voltage due to the different atomic mass. The mass ratio of the propellants is defined as

$$
\mathrm{MR}=\frac{M_{n}}{M_{\mathrm{Xe}}}
$$

where MR is the mass ratio of the propellant to xenon. The thrust produced by a thruster is defined as $F=u_{e} \dot{m}$. Assuming that ions of any species are accelerated through the same voltage, the exhaust velocity will be

$$
u_{e}=\sqrt{\frac{2 q_{i} V_{a}}{M_{\mathrm{Xe}}}} \sqrt{\frac{1}{\mathrm{MR}}}=\left(u_{e}\right)_{\mathrm{Xe}} \sqrt{\frac{1}{\mathrm{MR}}}
$$

and the thrust force is

$$
F=\left(u_{e}\right)_{\mathrm{Xe}} \dot{m} \sqrt{\frac{1}{\mathrm{MR}}}
$$

which is the thrust force of a xenon thruster divided by the square root of the mass ratio. Mass flows may either be the same between different propellants, or they may be adjusted so the same number of moles of propellant are used per second. In the case where mass flow is constant, $\dot{m}$ will be the same for all propellant options. In the case where molar flow is constant, the mass flow will change according to MR, while the discharge current will remain approximately constant. If the mass ratio is adjusted so molar flow rate and thus thruster current is constant, then the thrust will be the thrust of a xenon thruster multiplied by the square root of the mass ratio. The trends in thrust for different propellant ion masses are plotted in Fig. $1 . I_{\mathrm{sp}}$ under constant discharge voltage will be modified similarly to exhaust velocity. As $I_{\mathrm{sp}}$ is given by the equation

\footnotetext{
蛙Data available on-line at http://www.vngas.com/pdf/g54.pdf (xenon) and http://msds.ehs.cornell.edu/msds/msdsdod/a481/m240131.htm (krypton) [cited June 2005].

${ }^{\S}$ Data available on-line at http://msds.ehs.cornell.edu/msds/msdsdod/ a96/m47935.htm [cited June 2005].
} 


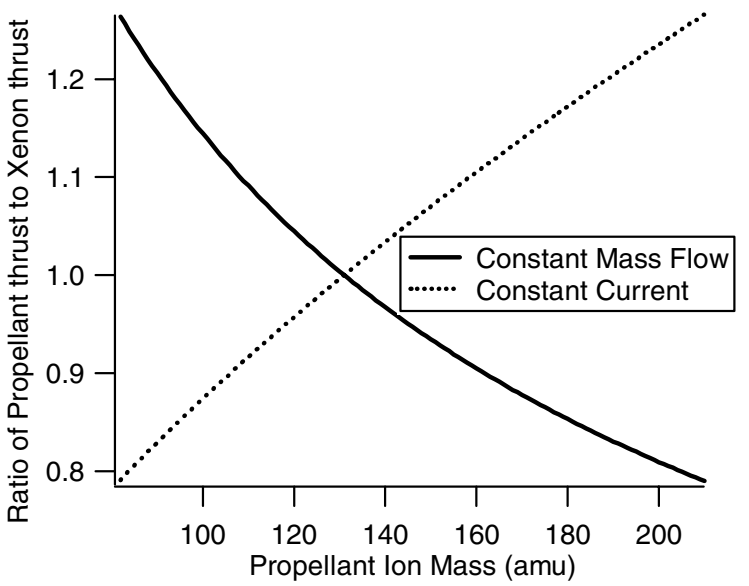

Fig. 1 Change in thrust vs propellant ion mass, assuming constant acceleration voltage.

$$
I_{\mathrm{sp}}=\frac{u_{e}}{g}
$$

$I_{\text {sp }}$ for any propellant is equal to the value for a xenon thruster, divided by the square root of the mass ratio. So a thruster that would normally operate on xenon at $2000 \mathrm{~s} I_{\mathrm{sp}}$ would operate on krypton at $2500 \mathrm{~s}$, and on bismuth at $1583 \mathrm{~s}$ for the same discharge voltage, with the other propellant options falling between the krypton and bismuth specific impulses. To maintain the same $I_{\mathrm{sp}}$ with different propellants, the discharge voltage would need to be modified. Large variations in the discharge voltage (outside the 200-600 V range of current flight thrusters) cause complications in plasma acceleration physics that may preclude the use of heavy atoms at high $I_{\mathrm{sp}}$, or light atoms at low $I_{\mathrm{sp}}$.

Aside from the exhaust kinetics, the choice of propellant has a direct influence on thruster efficiency. Ionization production represents a fundamental source of inefficiency, because any energy spent in creating an ion from a neutral is not available for conversion to beam kinetic energy. As the ionization energy is exhibited as a direct power loss from the thruster, keeping the ionization energy at a minimum will increase thruster efficiency. Whereas ionization energy is typically expressed as the energy required to singly ionize one neutral atom (eV/atom), a more convenient form for thruster analysis is the amount of energy required per kilogram of mass flow. This factor, $E_{\text {ion }}$, then accounts for the difference in ionization potentials as well as the difference in atomic masses of the candidate species, and can be calculated by dividing the ionization energy by the mass of an atom. $E_{\text {ion }}$ can then be employed in the equation

$$
P_{\text {ion }}=\dot{m} E_{\text {ion }}
$$

We can use $P_{\text {ion }}$ to calculate the maximum theoretical efficiency of an acceleration process assuming that the only energy loss is propellant ionization. Expressing the kinetic power in the exhaust beam as

$$
P_{\mathrm{kin}}=\frac{1}{2} \dot{m} u_{e}^{2}=\frac{\dot{m} g^{2} I_{\mathrm{sp}}^{2}}{2}
$$

Combining Eq. (ㅁ) with Eq. (ㅁ) yields

$$
\frac{P_{\text {ion }}}{P_{\text {kin }}}=\frac{2 E_{\text {ion }}}{g^{2} I_{\mathrm{sp}}^{2}}
$$

Eq. (7) is then the theoretical minimum efficiency penalty required to singly ionize and accelerate the propellant as a function of $I_{\mathrm{sp}}$. These ratios are plotted in Fig. 2.

The propellants with lower ionization energy-per-mass $\left(E_{\text {ion }}\right)$ require a smaller fraction of the total thruster power to ionize the propellant. It is also interesting to note that the difference between propellants becomes nearly insignificant at high $I_{\mathrm{sp}}$. At $1000 \mathrm{~s}$, the fractions range from approximately $6 \%$ for cesium up to $34 \%$ for

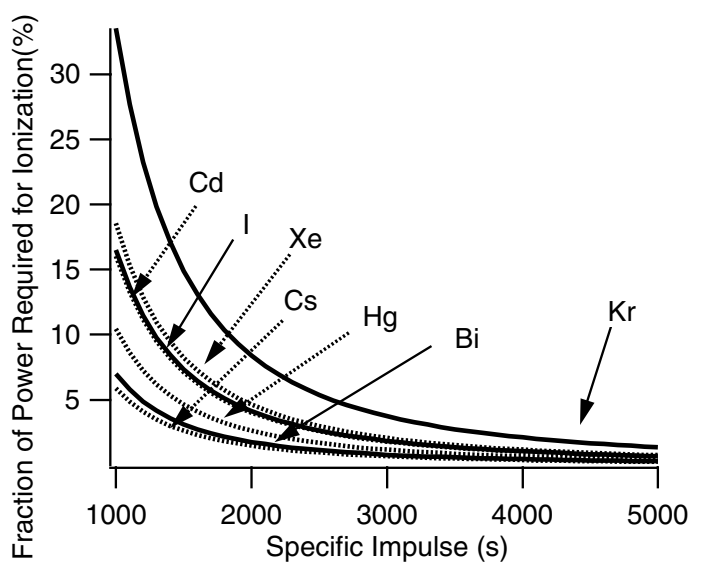

Fig. 2 Fraction of thruster power required for ionization vs $I_{\mathrm{sp}}$ for all propellants examined.

krypton, whereas at $5000 \mathrm{~s}$ no propellant option requires significantly more than $1 \%$ of total thruster power (krypton is highest at $1.3 \%$ ). It should be noted that these calculations are a theoretical minimum where all of the propellant is ionized, there are no doubly-charged ions, no energy is spent promoting atoms or ions to excited states, and all of the ions are produced only once; there is no wall recombination and reionization. Any reionization process will present an energy loss as an ion is essentially being created twice, requiring twice the energy expenditure. The ionization power will differ in a real thruster; however, the relative scaling between propellants should remain similar.

\section{B. Collision Considerations}

Any neutral propellant that is not ionized within the discharge chamber before escaping the thruster internal volume represents inefficiency in propellant utilization (it is not electrostatically accelerated and contributes negligibly to thrust). The probability of ionization for a given atom subject to an electron collision is given by the ionization cross section. These cross sections are dependent on the energy of the impacting (ionizing) electron and the atomic structure of the propellant atom. Experimentally determined ionization cross sections for bismuth [11], cesium [12,13], iodine [14], krypton [15], mercury [16], and xenon [15] were available in literature. Cross section measurements for cadmium could not be found. A comparison of xenon with the other propellants is plotted in Fig. 3 .

In general, the lower the atom's ionization energy the larger the ionization cross section. Thus cesium shows itself to be the most amenable to ionization, due to the extremely low ionization energy. However, bismuth and mercury do not have significantly lower cross sections than cesium. The smallest cross sections calculated were for the two propellants currently in use: xenon and krypton. Thus any change from xenon or krypton to another propellant discussed here will result in an increased probability of ionization within the discharge chamber.

Using the ionization cross section, the rate of ionizing collisions can be calculated. The rate of ionizing collisions is given by [17]

$$
v=\bar{\sigma} n_{e} \sqrt{\bar{v}_{n}^{2}+\bar{v}_{e}^{2}}
$$

To calculate estimates of $v$ the electron velocity distribution function was calculated based on an assumed $20 \mathrm{eV}$ temperature and the Maxwellian distribution [18]. The average electron-impact ionization cross section was calculated by integrating the product of the electron energy distribution function and the ionization cross section. $\bar{\sigma}$ is estimated assuming the propellant atoms are emitted with a thermal velocity distribution in equilibrium with the anode/ diffuser temperature. Average electron velocity is estimated similarly, assuming the temperature of the electrons is $20 \mathrm{eV}$. Given typical anode temperatures of $700^{\circ} \mathrm{C}$, the neutral velocity will be in the range of $300-500 \mathrm{~m} / \mathrm{s}$ for all species. Because the average 


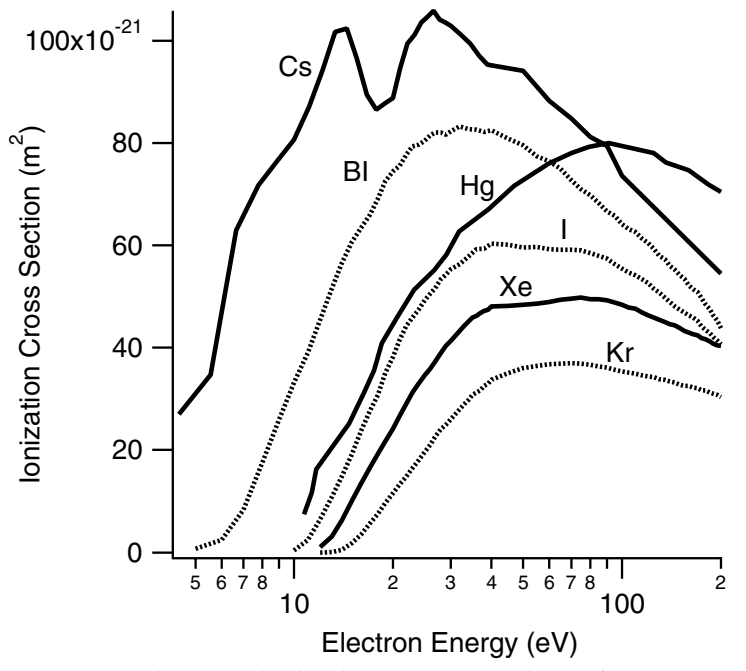

Fig. 3 Electron-impact ionization cross sections for the atoms examined between threshold and $200 \mathrm{eV}$.

electron velocity is 4 orders of magnitude higher, the neutral velocity can be neglected. Thus, collision frequencies will scale simply with ionization cross section and electron density. An electron number density of $2 \times 10^{18} \mathrm{~m}^{-3}$ was measured by Haas and Gallimore [18] and is used here as a representative value to calculate collision frequencies that may be typical within a Hall thruster discharge. Of critical importance to determination of the propellant use of a thruster is the electron temperature in the discharge channel. As it is impossible to determine the exact effect switching to another propellant will have on the electron temperature, values of electron temperature measured in xenon thrusters are used here for calculations. Calculated values of the collision frequency are in Table 2 .

Whereas the trends in collision frequency among the propellant candidates simply mirror the cross section scaling of Fig. $\underline{3}$, the magnitude of $v$ becomes particularly important in calculating the propellant utilization efficiency. To measurably contribute to the thrust, a propellant atom must be ionized before it is permitted to escape the discharge chamber. Thus if the residence time is defined as $t_{r}=L / v_{n}$ and $v_{n}$ is defined as

$$
\bar{v}_{n}=\sqrt{\frac{k T}{2 \pi m}}
$$

the factor $v t_{r}$ gives the ratio of residence time to collision time. Physically, this factor represents the average number of ionizing collisions experienced by a propellant atom before diffusing out of a discharge chamber of length $L$. If this factor is low, it is probable that propellant atoms will escape the discharge without being ionized. Using the calculated collision frequencies, assuming neutral atoms leave the gas diffuser with a temperature of $700^{\circ} \mathrm{C}$, and assuming the neutral atoms must travel $100 \mathrm{~mm}$ to leave the channel, the average number of ionizing collisions for each propellant species is shown in Table 2 .

Whereas the gross simplification used in determining the number of collisions precludes any confidence in the absolute magnitude of the numbers, the relative trends between propellants is a reliable indicator of utilization. The calculated ionizing collision rates show significant differences between species. When compounded with the
Table 3 First and second ionization energies

\begin{tabular}{lcc}
\hline \hline Propellant & First ionization energy, eV & Second ionization energy, eV \\
\hline Bismuth & 7.3 & 16.7 \\
Cadmium & 9.0 & 16.9 \\
Cesium & 3.9 & 23.2 \\
Iodine & 10.4 & 19.1 \\
Krypton & 14.0 & 24.4 \\
Mercury & 10.4 & 18.8 \\
Xenon & 12.1 & 21.2 \\
\hline
\end{tabular}

neutral diffusion velocity, some propellants show a much higher utilization than others. Krypton suffers here, as it has the lowest ionization cross section and the highest neutral diffusion velocity. Cesium, as expected, will experience the most ionizing collisions due to its very high ionization cross section. Of particular interest here, however, is bismuth, with an estimated number of collisions only slightly less than cesium. This is due in large part to the large cross section and extremely low neutral diffusion velocity of bismuth.

Whereas it is important to have an electron population with enough energy to ionize the propellant, it is equally important not to have an electron population so energetic as to produce significant amounts of multiply charged ions. Previous studies on xenon thrusters have given maximum electron temperatures above $15 \mathrm{eV}$ $[19,20]$. Probe-based studies of a $5-\mathrm{kW}$ thruster at several operating points show the temperature is dependent on flow rate, and can be nearly $30 \mathrm{eV}$ in low mass flow conditions [18]. Higher mass flows appear to cool the electrons, as the drifting electrons cannot acquire as much energy through "falling" towards the thruster anode after a collision. Any population of electrons with temperature greater than the second ionization potential of the propellant may create multiply ionized propellant ions. Multiply charged ions represent an inefficient use of propulsive power. For instance, a doubly charged ion will contribute twice the discharge current (and hence, draw twice the power) as a singly charged ion; however, will only be accelerated to a velocity $\sqrt{2}$ times more than its single counterpart.

Formation of multiply charged ions may be an issue for some propellants as shown in Table $\underline{3}$. Xenon has the drawback of a high first ionization energy, but its second ionization energy is quite high $(21.2 \mathrm{eV})$; thus it does not readily produce $\mathrm{Xe}^{2+}$ in typical Hall thruster plasmas, with approximately $90 \%$ of the xenon ions singly charged [21]. Cesium and krypton also will not form large fractions of multiple ions. Other elements, such as bismuth and cadmium, may be sensitive to multiply charged ion efficiency losses in an electron population with temperatures similar to those seen in xenon devices.

\section{Analysis of Spacecraft Interactions for Bismuth, Xenon, and Krypton}

At this point, bismuth, xenon, and krypton will be chosen for further analysis. As bismuth is nearly as efficient as cesium, without the toxicity or reactivity drawbacks, it appears to be the best choice for a high-thrust, low- $I_{\mathrm{sp}}$ thruster. Krypton is chosen as an alternative only for high- $I_{\mathrm{sp}}$ operation, as it is too energetically costly to ionize to be useful in a low- $I_{\mathrm{sp}}$ thruster.

The sputter rate of a propellant is highly important to thruster lifetime, especially at high- $I_{\mathrm{sp}}$ when propellant ions have significant energy. Typically, the maximum lifetime of the thruster is determined by the time required for sputter erosion to wear through the discharge channel walls and start eroding the electromagnet

Table 2 Number of ionizing collisions experienced by propellant atoms in a 100-mm channel

\begin{tabular}{lccc}
\hline \hline Propellant & Collision frequency, $\times 10^{2} \mathrm{~Hz}$ & Neutral diffusion velocity, m/s & Number of collisions \\
\hline Bismuth & 4.1 & 161 & 26 \\
Cesium & 5.5 & 202 & 32 \\
Iodine & 2.7 & 207 & 18 \\
Krypton & 1.4 & 254 & 6 \\
Mercury & 3.3 & 165 & 20 \\
Xenon & 2.1 & 203 & 10 \\
\hline \hline
\end{tabular}


poles. The sputter yield of ions normally incident on a solid surface can be calculated by [22]

$$
Y(E)=0.42 \frac{\alpha_{s} Q_{s} S_{n}(E)}{U_{0}\left[1+0.35 U_{0} S_{e}(\epsilon)\right]}\left[1-\sqrt{\frac{E_{\mathrm{th}}}{E}}\right]^{2.8}
$$

Of particular interest is $E_{\text {th }}$, as sputtering does not occur when incident ions have energies below $E_{\mathrm{th}}$. The sputtering threshold energy is calculated by

$$
E_{\mathrm{th}}=\left(\frac{4}{3}\right) \frac{U_{0}}{\gamma}
$$

$\gamma$ is involved in the calculation of the threshold sputtering energy, and thus affects the sputtering rate at all energies. $\gamma$ is calculated by the equation

$$
\gamma=\frac{4 M_{i} M_{s}}{\left(M_{i}+M_{s}\right)^{2}}
$$

Calculations were made for bismuth, krypton, and xenon ions incident on a carbon surface as a representative thruster material (it is unclear whether or how this model can be applied to BN as a target material). Whereas carbon is not normally used in Hall thrusters, there has been experimental effort into using it as an electrode material $[23,24]$

The sputtering threshold for ions on carbon was found to be different for each species: $202 \mathrm{eV}$ for bismuth, $135 \mathrm{eV}$ for xenon, and $95 \mathrm{eV}$ for krypton. These results mean that for a given distribution of ion energies, fewer bismuth ions will be above the sputtering threshold than xenon or krypton. For ions above threshold, the model can be used to predict sputter rates. The calculated sputter rates over a range of ion energies are plotted in Fig. 4 .

The results of sputtering calculations show that bismuth will induce less sputtering on carbon substrates at energies anticipated in Hall thruster operation. At high energies (greater than $1200 \mathrm{eV}$ ) bismuth will cause higher sputtering than xenon and krypton; however, this range is beyond the operational envelope of state-ofthe-art Hall thrusters. Bismuth's erosion advantage is further extended if sputtering is analyzed under constant mass flow. As bismuth has an atomic mass of $209 \mathrm{amu}$, nearly $60 \%$ greater than the atomic mass of xenon (131 amu) and 2.5 times as high as krypton (84 amu), a given mass flow of bismuth will contain fewer ions. To determine the effect of the increased mass of bismuth, the calculations were scaled to indicate the mass of surface sputtered away per unit mass of ions. These results are displayed in Fig. $\underline{5}$ and show that bismuth will cause less erosion than xenon or krypton given equal mass flows. These results are encouraging for the lifetime of a bismuth thruster, as the thruster structures should exhibit lower erosion for the same total mass throughput. The erosion rate for bismuth remains less than xenon or krypton until the ion energies approach $10,000 \mathrm{eV}$, at which point the erosion rates converge. For

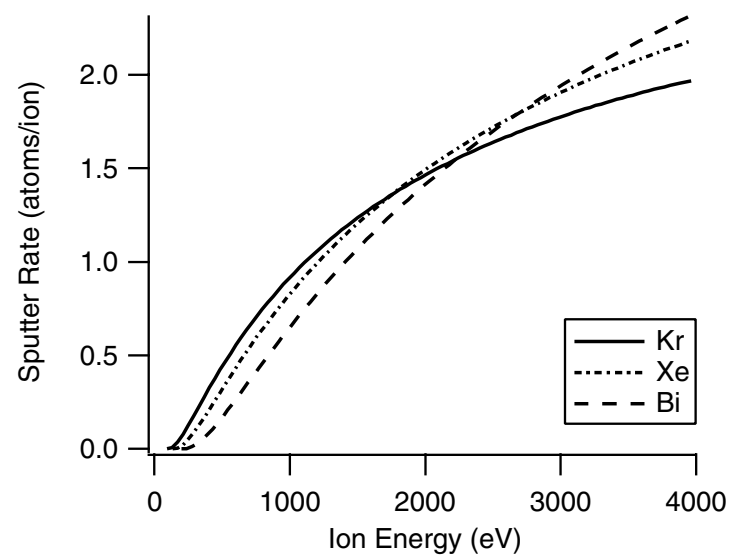

Fig. 4 Sputter yield of carbon under bismuth, xenon, and krypton ion collisions.

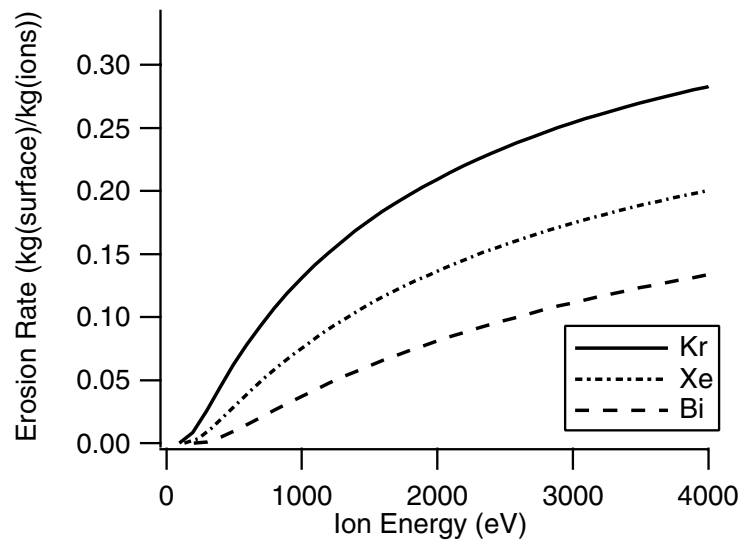

Fig. 5 Erosion rate of carbon per kilogram of bismuth, xenon, and krypton ions.

ion energies higher than $10,000 \mathrm{eV}$, which are not included in Fig. 5, the lighter propellants become favorable. It should be noted that the erosion rate is primarily driven by the ion mass and atomic number, and the ratios of these to the atoms in the surface. As the other propellants analyzed fall between krypton and bismuth in size, they will exhibit sputter rates somewhere between those of krypton and bismuth.

One complicating factor for use of bismuth or any other condensable propellant is deposition on spacecraft surfaces. As the surfaces of a spacecraft may be well below the melting point of a condensable propellant, ions impacting on spacecraft surfaces will stick similarly to the interior of a vacuum tank. This would present a problem for solar arrays and other optical systems, as it is not desirable to have coatings of opaque metals on the transparent lenses and plates of such systems. Other spacecraft integration issues include coating of radiator and dielectric surfaces. Coatings of propellant atoms on radiators will reduce emissivity and radiative efficiency. Dielectrics are very vulnerable to propellant deposition, as conductive coatings on the surface of a dielectric will render it ineffective. Spacecraft design and interaction studies will require future research.

\section{Feed System for Condensible Propellant Operation}

Development of a feed system for a condensible propellant is a major technical obstacle. A large advantage to mercury, as used in NASA thrusters, was that it was a liquid at room temperature; it could be transported through propellant lines with little heat input and the only significant need for external heating was for the evaporator [25]. This system may not be practical for bismuth or the other condensible propellants analyzed here, as the entire propellant feed system would need to be maintained above the melting point of the propellant. This would not present much of a problem for cesium due to the very low melting point, but for bismuth operation, the propellant must be maintained in excess of $271^{\circ} \mathrm{C}$. Bismuth thrusters in Soviet research used a propellant feed system that was maintained in excess of $1000^{\circ} \mathrm{C}$, to flow gaseous bismuth directly into the thruster [9]. Additionally, the thruster body was heated above $1000^{\circ} \mathrm{C}$ using resistive heaters. This method may present a problem, however, in that a number of heaters consuming significant amounts of power are likely required. Whereas using large amounts of power for a propellant evaporation system is possible in ground testing, any development of a flight system cannot allow for such expenditures. The use of condensible metal propellants in future Hall thrusters will require development of an energy-efficient feed system. An additional energy expenditure is the evaporation of the propellant, requiring enough heat input to overcome the heat of fusion of the propellant. The energy required to overcome the heat of fusion of the propellant is negligible compared to the total thruster power; Bismuth, for example, requires $54 \mathrm{MW}$ of power per $\mathrm{mg} / \mathrm{s}$ of mass flow to evaporate. 


\section{Conclusions}

For high- $I_{\mathrm{sp}}$ missions, krypton may prove advantageous as it provides the highest $I_{\mathrm{sp}}$ of the options presented here for a given acceleration voltage, and at such high exhaust energies the large ionization cost is minimized. The erosion rate due to sputtering will also be lower for krypton than other propellants at very high energies (above 10,000 eV). For high-thrust, low- $I_{\mathrm{sp}}$ missions, however, the large heavy atoms may provide a benefit. Bismuth provides higher thrust per unit mass than the lighter propellant options given equal accelerating voltages and is very easy to ionize. Whereas cesium is the easiest to ionize, once the average number of collisions in a Hall thruster channel is analyzed, it proves to be only incrementally better than bismuth. Cesium is also only marginally better in the fraction of thruster power required for ionization than bismuth. Heavy atoms also provide an advantage in erosion rate for most ion energies, up to extremely high specific impulses.

Condensible propellants also offer significant advantages over gases. Primary of these is the elimination of the costly and complex pumping apparatus required to maintain acceptable vacuum levels. This benefit is complementary to the increased efficiency of condensable species for low- $I_{\text {sp }}$, and correspondingly high flow rate, missions that are most expensive to ground test. Condensible propellants may cause spacecraft contamination issues, as unlike the gaseous propellants condensibles will deposit on spacecraft surfaces.

Among the condensible propellant options, bismuth shows the most promise for Hall thruster use and is likely superior to other candidates for high-thrust, low- $I_{\mathrm{sp}}$ missions. It combines the advantages of higher thrust at the same discharge current as a xenon thruster, low cost, ease of ionization, and lower sputter erosion rate than the other propellants examined. The main disadvantage to bismuth, however, is that any thruster design must incorporate a method of heating the bismuth evaporator to temperatures where evaporation is significant. It is also much less likely that spacecraft surfaces will be warm enough to avoid or reduce bismuth deposition due to the high melting and boiling points. A second, and possibly significant, disadvantage of bismuth may be its propensity to form doubly charged ions at lower electron temperatures than xenon.

\section{References}

[1] Kim, V., Popov, G., Kozlov, V., Skrylnikov, A., and Grdlichko, D., "Investigation of SPT Performance and Particularities of its Operation with $\mathrm{Kr}$ and $\mathrm{Kr} / \mathrm{Xe}$ Mixtures," IEPC Paper 01-065, Oct. 1997.

[2] Walker, M. R., "Effects of Facility Backpressure on the Performance and Plume of a Hall Thruster," Ph.D. Dissertation, Univ. of Michigan, Ann Arbor, MI, 2004.

[3] Grishin, S. D., Erofeev, V. S., Zharinov, A. V., Naumkin, V. P., and Safronov, I. N., "Characteristics of a Two-Stage Ion Accelerator with an Anode Layer," Zhurnal Prikladnoi Mekhaniki i Tekhnicheskoi Fiziki, Vol. 2, March-April 1978, pp. 28-36.

[4] Tverdokhlebov, O. S., and Semenkin, A. V., "Iodine Propellant for Electric Propulsion-To Be or Not To Be," AIAA Paper 2001-3350, July 2001.

[5] Bugrova, A. I., Kim, V., Maslennikov, N. A., and Morozov, A. I., "Physical Processes and Characteristics of Stationary Plasma Thrusters with Closed Electrons Drift," IEPC Paper 91-079, 1991.

[6] Dressler, R. A., Chiu, Y.-H., and Levandier, D. J., "Propellant Alternatives for Ion and Hall Effect Thrusters," AIAA Paper 2000-
0602, Jan. 2000.

[7] Kerslake, W. R., and Ignaczak, L. R., "Development and Flight History of SERT II Spacecraft," Journal of Spacecraft and Rockets, Vol. 30, No. 3, 1993, p. 258.

[8] Sovey, J. S., Rawlin, V. K., and Patterson, M. J., “A Synopsis of Ion Propulsion Development Projects in the United States: SERT I to Deep Space I," AIAA Paper 99-2270, June 1999; also NASA TM-1999209439.

[9] Tverdokhlebov, S., Semenkin, A., and Polk, J., "Bismuth Propellant Option for Very High Power TAL Thruster," AIAA Paper 2002-0348, Jan. 2002.

[10] Massey, D. R., King, L. B., and Makela, J. M., "Progress on the Development of a Direct Evaporation Bismuth Hall Thruster," AIAA Paper 2005-4232, 2005.

[11] Freund, R. S., Wetzel, R. C., Shul, R. J., and Hayes, T. R., "CrossSection Measurements for Electron-Impact Ionization of Atoms," Physical Review A, Vol. 41, No. 7, 1990, p. 3575.

[12] Nygaard, K. J., "Electron-Impact Ionization Cross Section in Cesium," Journal of Chemical Physics, Vol. 49, No. 5, 1968, p. 1995.

[13] McFarland, R. H., and Kinney, J. D., "Absolute Cross Sections of Lithium and Other Alkali Metal Atoms for Ionization by Electrons," Physical Review, Vol. 137, No. 4A, 1965, p. A1058.

[14] Hayes, T. R., Wetzel, R. C., and Freund, R. S., "Absolute ElectronImpact-Ionization Cross-Section Measurements of the Halogen Atoms," Physical Review A, Vol. 35, No. 2, 1987, p. 578.

[15] Wetzel, R. C., Baiocchi, F. A., Hayes, T. R., and Freund, R. S., "Absolute Cross Sections for Electron-Impact Ionization of the Rare Gas Atoms by the Fast-Neutral-Beam Method," Physical Review A, Vol. 35, No. 2, 1987, p. 559.

[16] Kieffer, L. J., and Dunn, G. H., "Electron Impact Ionization CrossSection Data for Atoms, Atomic Ions, and Diatomic Molecules: 1. Experimental Data," Reviews of Modern Physics, Vol. 38, No. 1, 1966, p. 1.

[17] Gombosi, T. I., Gaskinetic Theory, Cambridge Univ. Press, Cambridge, England, 1994.

[18] Haas, J. M., and Gallimore, A. D., "Considerations on the Role of the Hall Current in a Laboratory-Model Thruster," AIAA Paper 20013507 , July 2001.

[19] Meezan, N. B., Hargus, W. A., and Cappelli, M. A., "Anomalous Electron Mobility in a Coaxial Hall Discharge Plasma," Physical Review E, Vol. 63, No. 2, Paper 026410.

[20] Bishaev, A. M., and Kim, V., "Local Plasma Properties in a HallCurrent Accelerator with an Extended Acceleration Zone," Soviet Physics Technical Physics, Vol. 23, No. 9, Sept. 1978, p. 1055.

[21] King, L. B., "Transport-Property and Mass Spectral Measurements in the Plasma Exhaust Plume of a Hall-Effect Space Propulsion System," Ph.D. Dissertation, Univ. of Michigan, Ann Arbor, MI, 1998.

[22] Nastasi, M., Mayer, J. W., and Hirvonen, J. K., Ion-Solid Interactions Fundamentals and Applications, Cambridge Univ. Press, Cambridge, England, 1996.

[23] Raitses, Y., and Fisch, N. J., "Parametric Investigations of a Nonconventional Hall Thruster," Physics of Plasmas, Vol. 8, No. 5, 2001, p. 2579.

[24] Raitses, Y., Keidar, M., Staack, D., and Fisch, N. J., "Effects of Segmented Electrode in Hall Current Plasma Thrusters," Journal of Applied Physics, Vol. 92, No. 9, 2002, p. 4906.

[25] Kerslake, William, R., "Design and Test of Porous-Tungsten Mercury Vaporizers," AIAA Paper 72-484, April 1972.
A. Gallimore Associate Editor 